\author{
Leandro Galves*, Stefan Miska*, \\ Evren Ozbayoglu*, Małgorzata Ziaja*
}

\title{
STUDY OF THE PERFORMANCE OF LCMS IN FRACTURED FORMATIONS
}

\section{INTRODUCTION}

Lost circulation has become a common problem over the past decades, with the rise of technologies that allowed for deeper and more complex wells. Throughout the years, this issue has been often underestimated and has not been studied enough quantitatively. Drilling fluid losses into fractured formations is a major challenge. Lost circulation treatments are widely applied to mitigate the losses; however, the effectiveness of these treatments is affected by different physical properties of the used lost circulation materials (LCM). In field practice, drilling engineers are forced to understand the types of mitigations measures they can take in the event lost circulation occurs. In this case, LCMs, i.e., granular, flocculated or fibrous solids are added to the fluid to seal fractures. For years, the industry has used more of a trial-and-error approach in choosing the LCMs than anything else. To our knowledge the estimated industry cost related to the loss of mud, treatment of the drilling fluid (to mitigate lost circulation), downtime, and lost tools in the Gulf of Mexico: $\$ 1$ billion per year. Worldwide: about $\$ 2$ to 4 billion year.

This paper presents a study of the effectiveness of LCMs to seal the natural formation fractures that allows better understanding of the phenomena that affect fluid loss during drilling. The LCMs seal effectiveness is defined by the fluid loss to the formation, durability, and resistance to the changes in differential pressure during drilling operations. First the theoretical models; one predicting LCMs bridging in a fracture, and

\footnotetext{
* The University of Tulsa, Drilling Research Projects
} 
second predicting LCMs seal fractability were developed. Then, an experimental facility to test LCM was constructed for the experimental work, where the behavior of such materials in reducing lost circulation was observed. The LCMs seal effectiveness as the effects of particle size distribution (PSD) of LCM, the concentration of LCM in the drilling fluid, the width of the fracture, the fracture inclination, and the fracture configuration were investigated. The results were analyzed with a computer simulator. Finally, this simulator is intended to serve as guidance for decision making on which LCM to use in a particular field situation.

\section{THEORETICAL MODELING}

In this study, the performance of the LCMs in sealing natural fractures was described by two models. The first accounts for the LCM bridging process to minimize fluid loss; the second, determines the fractability of the LCM seal.

\subsection{Model Development for LCM Application}

There are several factors that affect the formation of the LCM bridge, including fracture geometry, fracture roughness, drilling fluid rheology, LCM shape, LCM resiliency, LCM type, LCM resistance to shear, LCM concentration, LCM particle size distribution (PSD), and the number of fractures in the system. Under these circumstances, a stochastic model is suitable to compute the ideal concentration of LCM particles inside the drilling fluid that would allow for minimum losses. Moreover, this model can also give information about the time spent to seal the fracture, the location of the bridge, as well as the flow rate after its formation.

The model was developed for the following assumptions:

- Non-Newtonian fluid: Yield Power-Law model.

- Isothermal conditions inside the fracture.

- The volume of fluid is much larger than the volume of particles.

- Laminar flow within the fracture.

- The fracture is simplified to consist of a set of two parallel plates of infinite length.

- The thickness of the LCM bridge is constant once it is formed.

- The LCM seal begins from the wellbore radius $\left(R_{w}\right)$.

- Constant fracture width.

- Steady-state condition is reached once the LCM seal is formed: the flow rate in both LCM bridge and filtration region are equal. 
The following step-by-step algorithm was applied for calculations:

1) The system that is composed of drilling fluid and fracture is divided in grids. Each grid corresponds to the volume of an LCM particle.

2) The probability of the presence of an LCM particle in each grid is calculated per time, as well as the probability of plugging.

3 ) The probability of plugging is made equal to 1 . Then, we can find the time and concentration to achieve that.

4) The time and concentration to plug the fracture is calculated based on the particles size.

5) Once the plug time is found, the goal is to estimate the position of the fluid front $\left(R_{f}\right)$.

6) The pressure at the fluid front is calculated.

7) The value of the pressure in the interface between the LCM seal and the filtration region $\left(P_{m}\right)$, according to Figure 1 , is guessed [10].

8) The fluid flow rate in the filter cake region $\left(Q_{1}\right)$ and in the filtration region $\left(Q_{2}\right)$ are computed [10].

9) If $Q_{1}$ and $Q_{2}$ differ, step 6 is repeated all the way to 8 . Otherwise, the final flow rate is $Q_{1}[10]$.

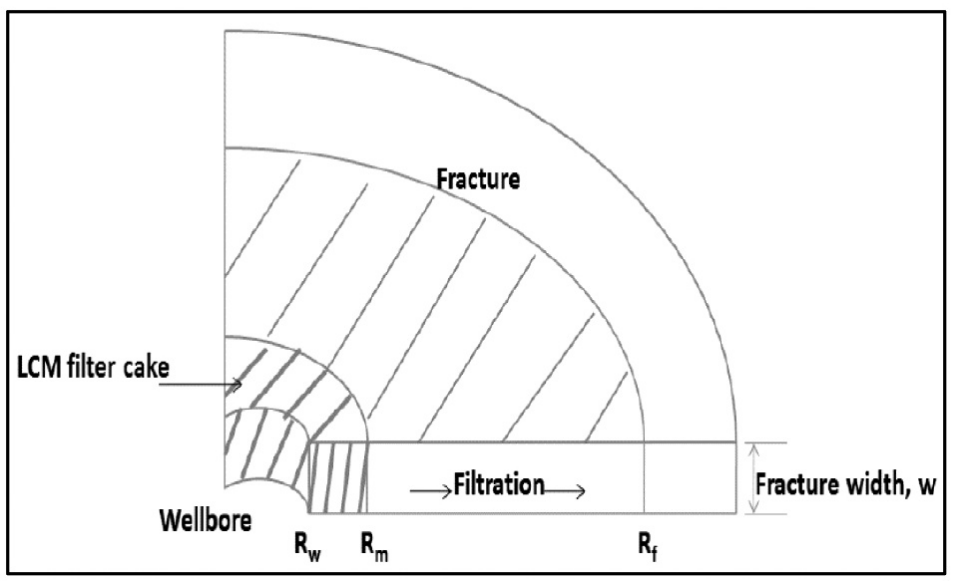

Fig. 1. Schematic of radial flow in a fracture with an LCM feal [10]

\section{Before the LCM bridge is formed}

The space between the plates simulating fracture is divided into grids. Each one has a volume equal to the volume of an LCM particle. The end goal is to compute the 
probability of finding a column of grids plugged by LCM particles. According to the probability theory, the random experiment is defined as: mix $n_{i}$ particles of LCM in $m_{i}$ grids before lost circulation starts. At time $t_{1}$, fluid starts flowing inside the fracture. The events related to this experiment are represented by the symbol $A_{l, c}^{t}$, which is defined as "there is a particle in the grid $(l, c)$ at time $t$ ". In this case, $l$ is the number of rows of grids in the fracture and $c$ is the number of columns.

For the flow of Yield Power-Law fluid in a slot, the average velocity is computed according to Mitchell and Miska [6]. Eq. (1) gives the flow rate of the fluid:

$$
q=\frac{2 w\left(\frac{h}{2}\right)^{2+\frac{1}{n}}\left(\frac{1}{K} \frac{d p_{f}}{d r}\right)^{\frac{1}{m}}\left(1-\frac{\tau_{y}}{\left(\frac{h}{2}\right)\left(\frac{d p_{f}}{d r}\right)}\right)^{1+\frac{1}{m}}\left(\frac{\tau_{y}}{\left(\frac{h}{2}\right)\left(\frac{d p_{f}}{d r}\right)}+1+\frac{1}{m}\right)}{\left(\frac{1}{m}+1\right)\left(\frac{1}{m}+2\right)}
$$

\section{After the LCM bridge is formed}

Once the LCM seal is formed, and steady-state is achieved, the problem is approached from a whole different angle. This part of the mathematical model is an improvement of the previous TUDRP model presented by [10]. It is based on the assumption that the LCM seal is a porous medium, and even after its formation there will persist a certain amount of fluid loss. Consequently, the seal will be as effective as the least permeable it is.

According to Zeng [10], by making the flow rate at the filter cake and filtration region equal, we can compute its value at two fronts: in the filter cake, or at the filtration region.

In the filter cake, the flow rate can be found considering a fluid flow through porous medium, according to Zeng [10]. The flow rate in the filtration region can be computed by Eq. (2), from Majidi [5]:

$$
q=\frac{(1-m) \frac{1}{m}\left(\frac{4 \pi m}{2 m+1}\left(\frac{w}{2}\right)^{2+\frac{1}{m}}\right)\left(\left(P_{f}-P_{m}\right)-\left(\frac{2 m+1}{m+1}\right)\left(\frac{2 \tau_{y}}{w}\right)\left(R_{f}-R_{w}\right)\right)^{\frac{1}{m}}}{\left(K\left(R_{f}{ }^{1-m}-R_{w}{ }^{1-m}\right)\right)^{\frac{1}{m}}}
$$


The modifications made in this model account for the fact that the fluid front radius is a function of time, and is given according to Majidi [5], in Eq. (3):

$$
\frac{d R_{f}}{d t}=\frac{w^{2}\left(\Delta P-\left(\frac{3 \tau_{y}}{w}\right)\left(R_{f}-R_{w}\right)\right)}{12 \mu_{p} R_{f} \ln \left(\frac{R_{f}}{R_{w}}\right)}
$$

With initial condition: as $t=0 \rightarrow R_{f}=R_{w}$.

Eq. (3) can be solved numerically. $\Delta P$ is computed at $R_{f}$ when steady-state fluid flow begins. Thus, it is a good approximation to say that $\Delta P=P_{w}-P_{f}$. To find the value of $P_{f}(t)$, according to the definition of isothermal compressibility:

$$
c=-\frac{1}{V}\left(\frac{\partial V}{\partial P}\right)_{T}
$$

The volume of fluid is:

$$
V=\left(L-R_{f}\right) A
$$

Solving for pressure of fluid front per time:

$$
P_{f}(t)=\frac{\ln \left(L-R_{w}\right)-\ln \left(L-R_{f}\right)}{c}+P_{0}
$$

Finally, the step-by-step algorithm can be followed as discussed above.

\subsection{Fractability model of the LCM seal}

Knowing that the performance of the LCM filter cake may depend on its stability and its breakdown pressure, geomechanical concepts were used to calculate the stresses in the LCM filter cake, as well as the fracture and the collapse pressures that might cause failure.

The fractability model was developed under the following assumptions:

- Filter cake can be considered a porous medium.

- Poro-elastic properties of a filter cake are the same as of formation drilled.

- Filter cake has a ring shape.

- Plane strain.

- No heat flow.

- Isotropic rocks. 
The strain state in the filter cake was determined from a force balance in a differential element of the filter cake as presented $\mathrm{d}$ in a free body diagram (FBD presented in Figure 2.

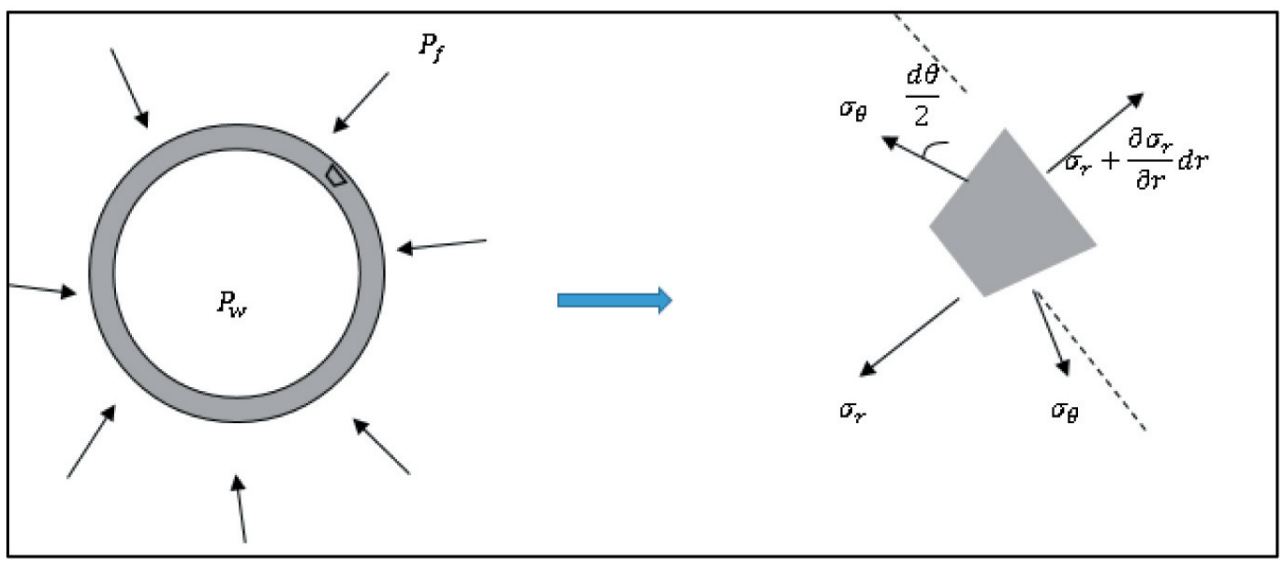

Fig. 2. FBD of an element of the LCM filter cake

The sum of the forces in the radial direction is:

$$
\left(\sigma_{r}+\frac{\partial \sigma_{r}}{\partial r} d r\right)(r+d r) d \theta-\sigma_{r} r d \theta-\sigma_{\theta} d r \sin \left(\frac{d \theta}{2}\right)-\sigma_{\theta} \sin \left(\frac{d \theta}{2}\right) d r=0
$$

Solution to Eq. (7) is given by:

$$
u(r)=K_{1} r+\frac{K_{2}}{r}-\frac{\alpha}{\lambda_{B}+2 G_{B}} \frac{1}{r} \int_{r_{w}}^{r} r^{\prime} P\left(r^{\prime}\right) d r^{\prime}
$$

Defining the following constants:

$$
\begin{array}{ll}
C_{1}=\frac{E_{B}}{\left(1+v_{B}\right)\left(1-2 v_{B}\right)}, & C_{2}=\frac{\alpha}{\lambda_{B}+2 G_{B}} \\
C_{3}=v_{B} \frac{\alpha}{\lambda_{B}+2 G_{B}}, & C_{4}=\frac{r_{w}{ }^{2} r_{f}{ }^{2}}{r_{f}{ }^{2}-r_{w}{ }^{2}}
\end{array}
$$


The boundary conditions are:

$$
B C 1: r=r_{w} \rightarrow \sigma_{r}\left(r_{w}\right)=P_{w}, \quad B C 2: r=r_{f} \rightarrow \sigma_{r}\left(r_{f}\right)=P_{f}
$$

The pore pressure for radial flow can be represented by Eq. (10):

$$
P(r)=P_{w}+\left(\frac{P_{f}-P_{w}}{\ln \left(\frac{r_{f}}{r_{w}}\right)}\right) \ln \left(\frac{r}{r_{w}}\right)
$$

The integration of Eq. (10) is solved by parts to obtain:

$$
\int P(r)=\left(\frac{P_{f}-P_{w}}{\ln \left(\frac{r_{f}}{r_{w}}\right)}\right)\left(\frac{r^{2}}{2} \ln \left(\frac{r}{r_{w}}\right)-\frac{r^{2}}{4}\right)+P_{w} \frac{r^{2}}{2}+C
$$

Finally, using Eq. (11), with the appropriate boundary conditions, $\sigma_{r}^{\prime}, \sigma_{\theta}^{\prime}$, and $\sigma_{z}^{\prime}$, (the stresses in the filter cake) at $r=r_{w}$ are obtained according to the Eqs. (12).

$$
\begin{aligned}
& \sigma_{r}^{\prime}=P_{w}-\alpha P_{w} \\
& \sigma_{\theta}^{\prime}=\left(\frac{2 C_{4}}{r_{w}{ }^{2}}+\frac{2 C_{4} C_{2} C_{1}}{r_{w}{ }^{2}}-\frac{2 C_{4} C_{3} C_{1}}{r_{w}{ }^{2}}\right) P_{f}+ \\
& +\left(1-\left(2 C_{4}\right) / r_{w}{ }^{2}-\left(2 C_{4} C_{2} C_{1}\right) / r_{w}{ }^{2}+\right. \\
& \left.+\left(2 C_{4} C_{3} C_{1}\right) / r_{w}{ }^{2}+C_{2} C_{1}-2 C_{3} C_{1}\right) P_{w}-\alpha P_{w} \\
& \sigma_{z}^{\prime}=\left(\frac{2 C_{4}}{r_{w}{ }^{2}}+\frac{2 C_{4} C_{2} C_{1}}{r_{w}{ }^{2}}-\frac{2 C_{4} C_{3} C_{1}}{r_{w}{ }^{2}}\right) v P_{f}+ \\
& +\left(\begin{array}{c}
2-\frac{2 C_{4}}{r_{w}{ }^{2}}-\frac{2 C_{4} C_{2} C_{1}}{r_{w}{ }^{2}}+\frac{2 C_{4} C_{3} C_{1}}{r_{w}{ }^{2}} \\
+C_{2} C_{1}-2 C_{3} C_{1}
\end{array}\right) P_{w}-\alpha P_{w}
\end{aligned}
$$


Considering that $\sigma_{r}^{\prime}, \sigma_{\theta}^{\prime}$, and $\sigma_{z}^{\prime}$ are the principal stresses, the Mohr-Coulomb and a tensile failure criterion are applied for two cases:

\section{Case 1: $\sigma_{\theta}{ }^{\prime}>\sigma_{z}{ }^{\prime}>\sigma_{r}{ }^{\prime}$}

The Mohr-Coulomb criterion becomes:

$$
\sigma_{\theta}^{\prime}=U C S+\sigma_{r}^{\prime} \tan ^{2} \beta
$$

Then, substituting appropriate Eq. (12) in Eq. (13) and solving for $P_{w}$, we obtain:

$$
P_{w \min }=\frac{U C S+\left(\frac{2 C_{4}}{r_{w}{ }^{2}}+\frac{2 C_{4} C_{2} C_{1}}{r_{w}{ }^{2}}-\frac{2 C_{4} C_{3} C_{1}}{r_{w}{ }^{2}}\right) \Delta P}{+1+C_{2} C_{1}-2 C_{3} C_{1}-\alpha-(1-\alpha) \tan ^{2} \beta}
$$

where $P_{f}=P_{w}-\Delta P$.

\section{Case 2: $\sigma_{z}{ }^{\prime}>\sigma_{\theta}{ }^{\prime}>\sigma_{r}{ }^{\prime}$}

Solving for $P_{w}$ :

$$
P_{w \min }=\frac{U C S+\left(\frac{2 C_{4}}{r_{w}{ }^{2}}+\frac{2 C_{4} C_{2} C_{1}}{r_{w}{ }^{2}}-\frac{2 C_{4} C_{3} C_{1}}{r_{w}{ }^{2}}\right) v \Delta P}{+2 v+v C_{2} C_{1}-2 v C_{3} C_{1}-\alpha-(1-\alpha) \tan ^{2} \beta}
$$

where $P_{f}=P_{w}-\Delta P$.

In case of tensile failure, $\sigma_{\theta}{ }^{\prime}$ was considered as the lowest principal stress. That is associated with patterns of vertical fractures. Substituting Eq. (12) in Eq. (14), and solving for $P_{w}$ :

$$
P_{w \max }=\frac{T_{0}+\left(\frac{2 C_{4}}{r_{w}{ }^{2}}+\frac{2 C_{4} C_{2} C_{1}}{r_{w}{ }^{2}}-\frac{2 C_{4} C_{3} C_{1}}{r_{w}{ }^{2}}\right) \Delta P}{+1+C_{2} C_{1}-2 C_{3} C_{1}-\alpha}
$$

where $P_{f}=P_{w}-\Delta P$.

An Excel ${ }^{\circledR}$ program was used to calculate the stresses in the LCM filter cake, the minimum, and the maximum pressures allowed inside the wellbore to avoid failure in the LCM filter cake. 
A sensitivity analysis of the equations for maximum and minimum pressures in the wellbore tested the influence of Poisson's ratio, Young's modulus, and pressure difference. Biot's coefficient was kept 1, because the LCM filter cake can be considered as highly unconsolidated.

Figure 3 shows the pressures are initially small in absolute values and exponentially increase as Poisson's ratio increases. The pressures go to infinity when the Poisson's ratio become 0.5. The higher Poisson's ratio, more difficult it is for the collapse or fracture of the LCM filter cake.

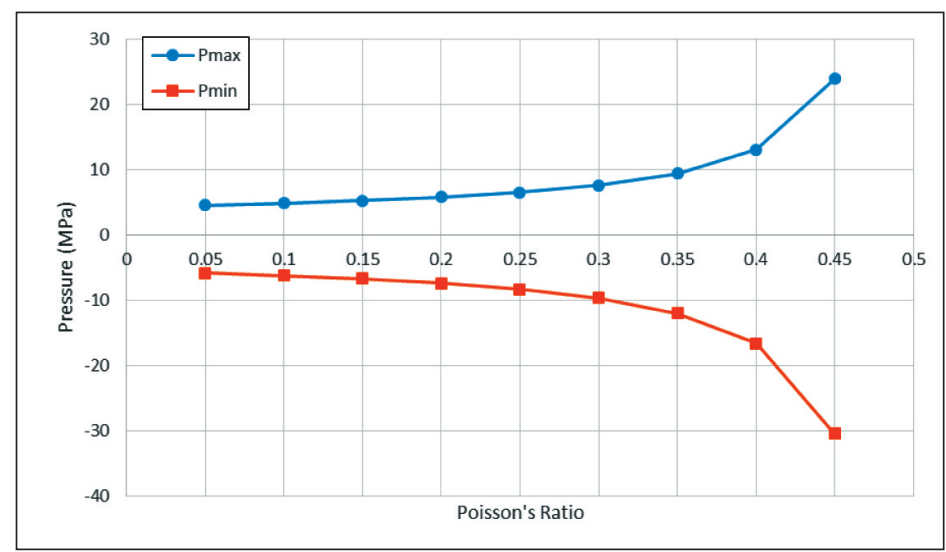

Fig. 3. Influence of Poisson's ratio in fracture and collapse pressures

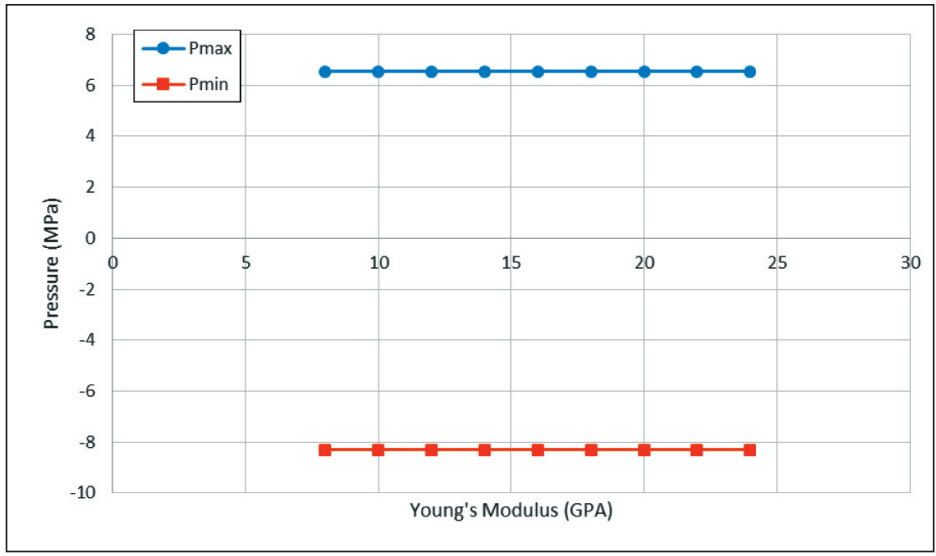

Fig. 4. Influence of Young's modulus in fracture and collapse pressures

Figure 4 shows that Young's modulus does not influence the maximum and minimum pressures that the LCM filter cake can withstand. 
Figure 5 shows that, on the one hand, the higher the pressure difference in the moment of formation of the LCM filter cake, the higher is the maximum pressure allowed in the wellbore. On the other hand, the increase in pressure difference results in an increase in the collapse pressure.

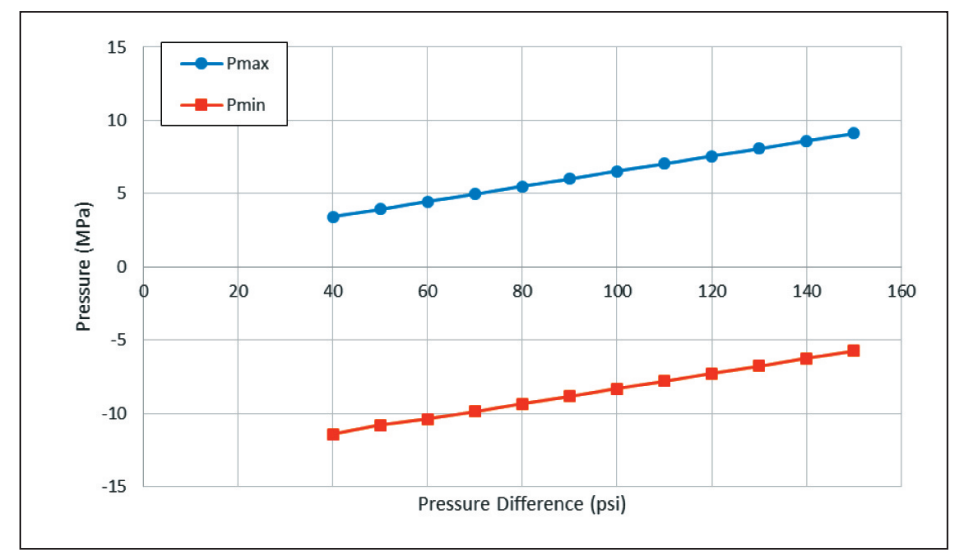

Fig. 5. Influence of the pressure difference between wellbore and formation fluid in the fracture and collapse pressures of the LCM filter cake

\section{EXPERIMENTAL STUDY}

The experimental setup was constructed to simulate a lost circulation in a fracture formation. The lost circulation facility was built inside the Fluid Characterization Lab, in the Dynamic Testing Facility (in TUDRP labs).

\subsection{Experimental setup}

A schematic of the experimental setup is presented in Figure 6.

The system is comprised of the following components:

- T1: tank where water is mixed with LCM. Its maximum capacity is 19 gallons.

- P1 and P2: AMT centrifugal pumps able to apply $25 \mathrm{HP}$ to the fluid. The circulation rate can be adjusted using a Hitachi variable frequency drive.

- Bypass: valve used to allow fluid circulation before the experiment actually starts.

- P: pressure gauges that measure the pressures before and after the limestone core.

- Limestone core: drilled using the TUDRP drilling rig. The core was cut in the middle with a rock cutting saw to simulate a fracture (Fig. 7). Two metal spacers were put between the core pieces to separate them and control the fracture aperture.

- T2: tank that collects fluid that is lost after the start of the experiment. 


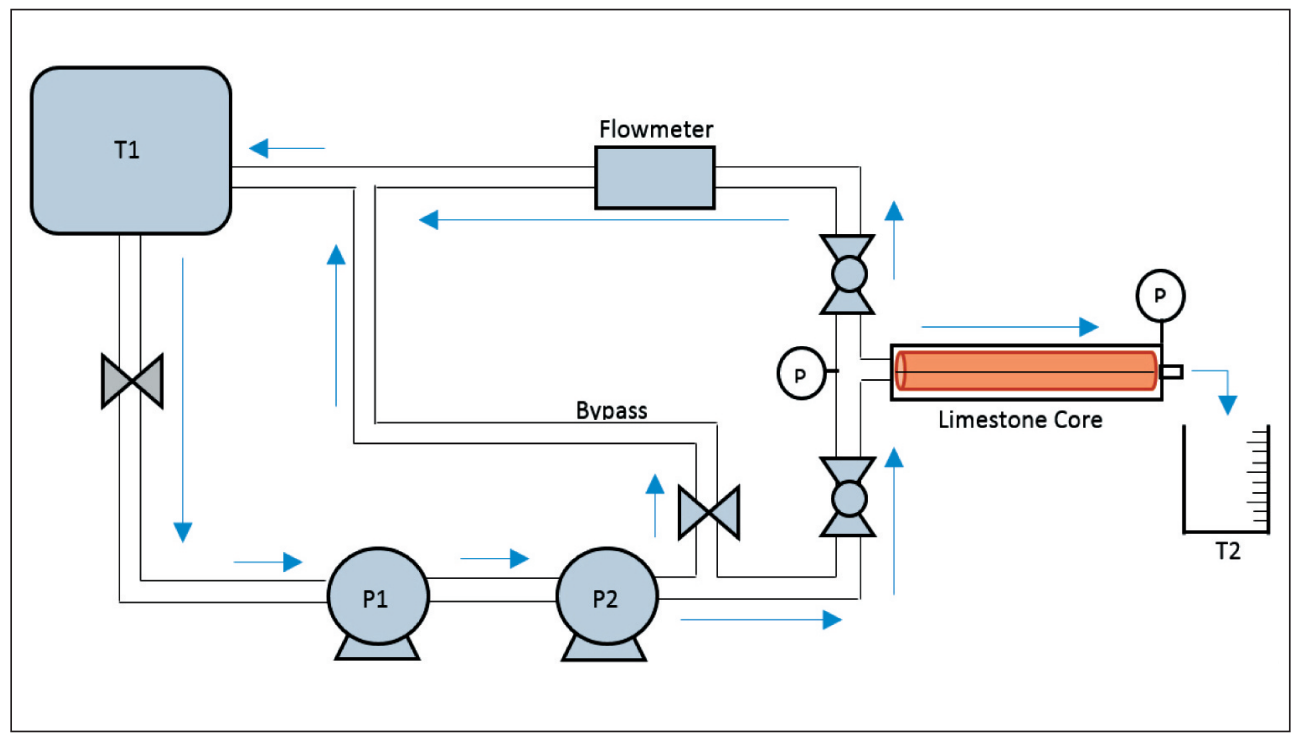

Fig. 6. Schematic of the experimental setup

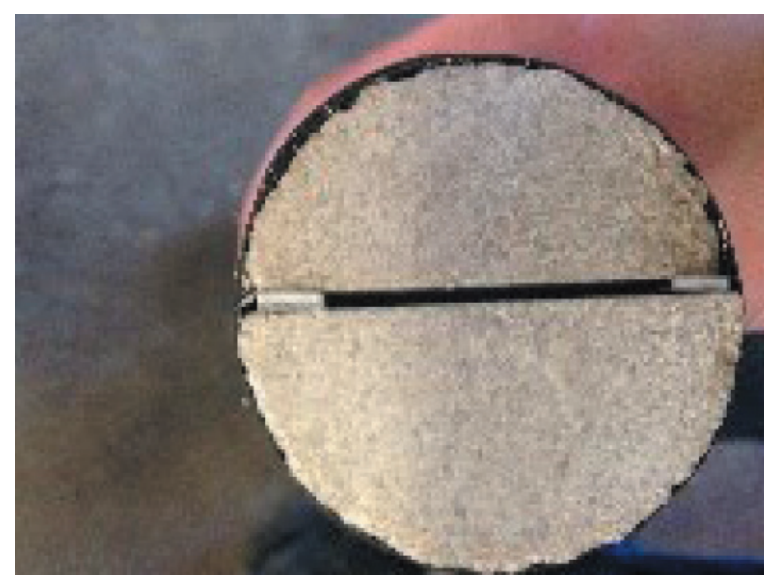

Fig. 7. Fracture in the limestone core

\subsection{Experimental results}

The two different LCMs were tested: walnut and a cellulosic granular material. The criterion used to stop the experiment was that each of the LCMs should either form a seal or correspond to a fluid loss of over 2,400 milliliters (ml) of fluid.

The test matrix is presented in Table 1 . 
Table 1

Test matrix

\begin{tabular}{|l|c|}
\hline \multicolumn{1}{|c|}{ Type of LCM (aspect ratio is 1$)$} & Walnut, cellulosic material \\
\hline Concentration $\left[\mathrm{kg} / \mathrm{m}^{3}\right]$ & $2.5,5,10,15,20$ \\
\hline Volume of the System $[\mathrm{gal}]$ & 18 \\
\hline Fracture Aperture $[\mathrm{mm}]$ & 1,3 \\
\hline Fracture Inclination (degrees) & $0,+25,-25$ \\
\hline Rock Type & Limestone \\
\hline Temperature $\left[{ }^{0} \mathrm{~F}\right]$ & 100 \\
\hline Apparent Viscosity at $600 \mathrm{rpm}[\mathrm{cp}]$ & 0 \\
\hline Yield Stress $\left[\mathrm{lb} / 100 \mathrm{ft}^{2}\right]$ & 60 \\
\hline Pressure Difference $[\mathrm{psi}]$ & Horizontal, vertical $1 \mathrm{~mm}$ frac), 70 (for $3 \mathrm{~mm}$ frac) \\
\hline Configuration & 0 \\
\hline
\end{tabular}

\section{LCM Concentration}

The concentration of LCM in the drilling fluid plays a major role in its ability to plug fractures. The very high concentrations lead to a fast plug but result in high wellbore pressure with all the negative consequences. On the other hand, too small concentrations, might not be sufficient to seal the fractures. As the width of fractures is difficult to predict, it is very important not only to determine the proper LCMs concentration but also the range of the PSD for the given wellbore situation.

As shown in Figure 8 the curves of flow rate per time obtained for three different walnuts' concentrations almost overlap for at all three concentrations tested. The LCMs plugged the fracture with $1 \mathrm{~mm}$ aperture mostly during the first 2 seconds. The seal formed in these experiments is shown in Figure 9. The plugs were relatively small in thickness and remained at the entrance of the fracture.

Figure 10 shows that the D90 of walnut is about the same size as the fracture aperture. In addition, as shown in Figure 10, the D50 is 1/3 of it. In the case of the cellulosic material, the majority of particles were too small, even though the D100 was $1 / 3$ of the fracture aperture.

Because of the undeniable importance of the D50 of LCM, the next set of experiments focused on testing the influence of different concentrations when the D50 was $1 / 3$ of the fracture aperture. The sizes remained between 297 micrometers $(\mu \mathrm{m})$ and $420 \mu \mathrm{m}$. 


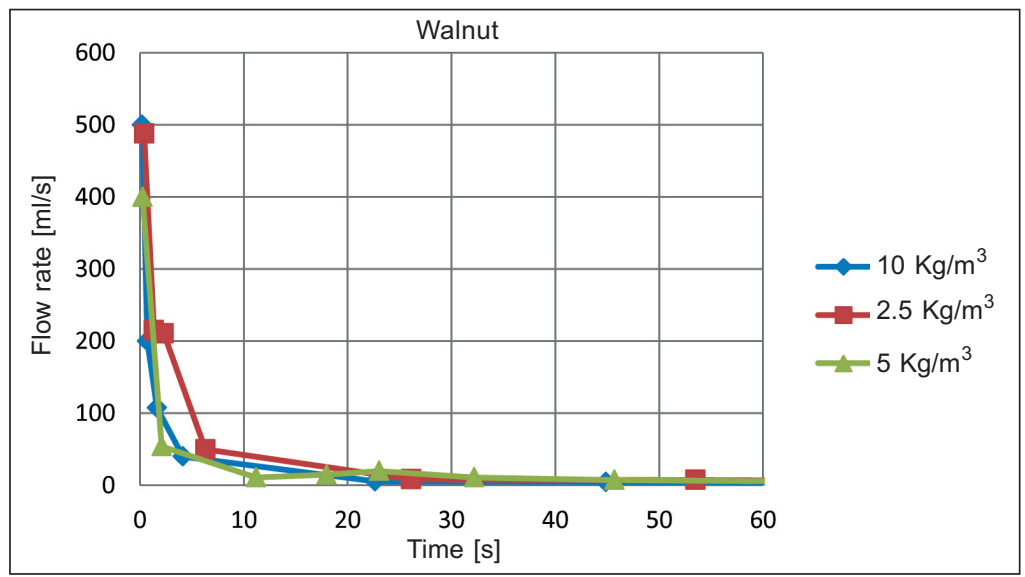

Fig. 8. Experiments with different particle concentrations of walnut

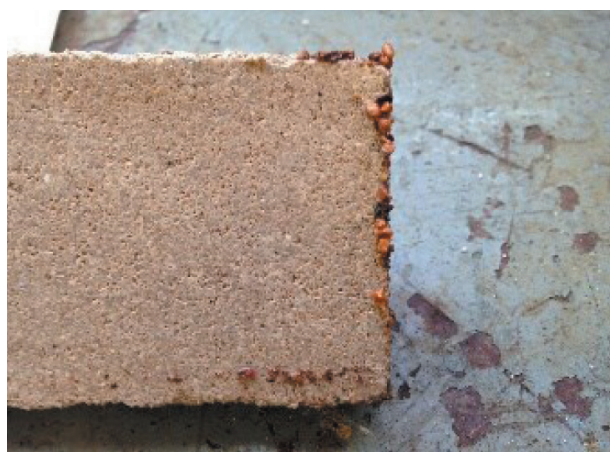

Fig. 9. Seal formed after each experiment presented in Figure 8

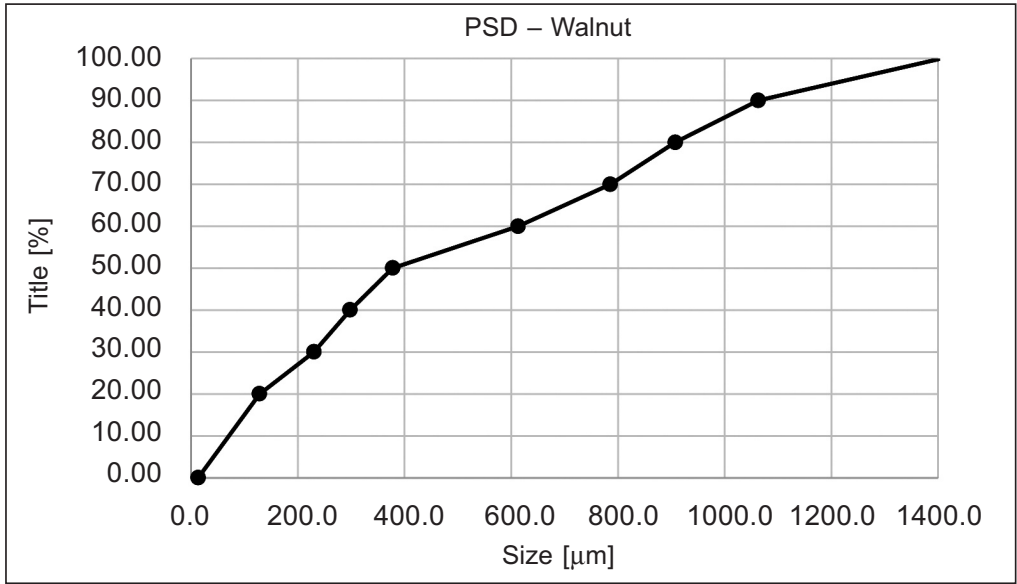

Fig. 10. The PSD of walnut corresponding to Figure 8 
When walnut $5 \mathrm{~kg} / \mathrm{m}^{3}$ was used, no plug was observed. The results for $10 \mathrm{~kg} / \mathrm{m}^{3}$ of walnut are shown in Figure 11. After about 90 seconds of the experiment, the flow rate reduced to almost zero. Thus, it is safe to say that for this concentration, the LCM plugged the fracture.

For higher concentrations of walnut, the fracture was also plugged, each time slightly earlier, 85 seconds and 56 seconds, respectively.

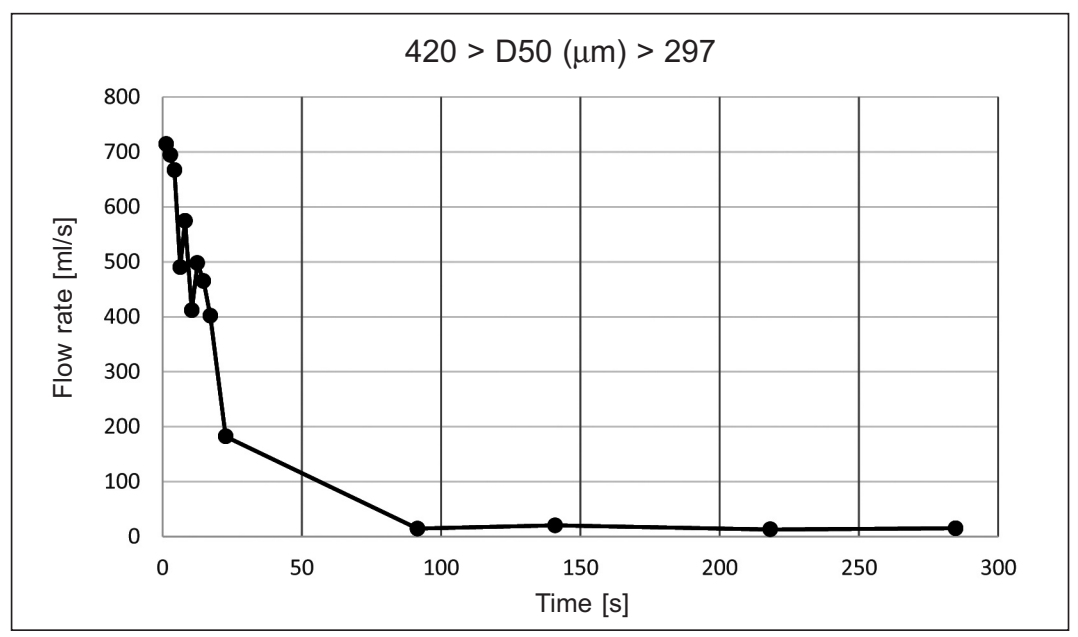

Fig. 11. Walnut at $10 \mathrm{~kg} / \mathrm{m}^{3}$ with size about $1 / 3$ of the fracture aperture

When the cellulosic material was used, none of the concentrations was sufficient to plug the fracture. In these cases, the particles that composed the cellulosic material were considerably smaller than the fracture aperture.

\section{LCM size}

The purpose of this set of experiments was to analyze the validity of the Bridging Theory. This theory states that a fracture will be plugged when the average size of LCMs ranges from $1 / 3$ to $1 / 7$ of the fracture opening. These particles tend to form an arc-shaped structure that blocks the fracture. Particles outside this range would not be able to stop the lost circulation.

Additionally, the oil industry has been making use of a large range of particle size distribution because of the uncertainties about the fracture opening. This experimental work clarifies the different effects of size, and may be used to narrow down the large-range PSD often adopted in the industry.

The influence of the LCM size in the flow rate of fluid lost in the fracture with aperture of 1 millimeter ( $\mathrm{mm}$ ) was tested experimentally. The size where the walnut particles were $1 / 3$ of the fracture opening presented the best results in terms of plugging the fracture faster and providing a stable seal (see Fig. 11). Upon a diminution in size, reduc- 
tion in flow rates could still be achieved, except when the size reduced to about $1 / 7$ of the fracture opening. In that case, no plug was observed.

Similar results were obtained for the fracture aperture $3 \mathrm{~mm}$ where the walnut particles having about $1 / 3$ of the fracture opening presented the best results in terms of plugging the fracture faster and providing a stable seal. The fracture could not be plugged for other sizes.

To conclude, the Bridging Theory provides an accurate prediction of the sizes that have to be used to plug a fracture; however, the larger the fracture, the less reliable the theory becomes.

\section{Fracture inclination}

The experimental tests showed that in case of positive values of inclination (against gravity), the fracture was plugged faster than for zero inclination. For negative values of inclination, the results did not show considerable difference. In both cases, $1 \mathrm{~mm}$ fractures were plugged in less than 7 seconds. However, when the size of the fracture increased to $3 \mathrm{~mm}$, the fracture could not be sealed.

\section{Fracture orientation}

The influence of the fracture orientation is also important. The vertical fractures are more likely to be formed in deep wells while the horizontal fractures, with radial flow, are more possible to occur in shallower formations.

As shown in Figure 12, the results obtained for the LCM with $10 \mathrm{~kg} / \mathrm{m}^{3}$ concen-tration applied in a $1 \mathrm{~mm}$ vertical fracture indicate that the flow rate of fluid lost presented reductions, but did not completely stop. A picture of the fracture opening in Figure 13 shows that the lower parts of the fracture were sealed, while the top remained open.

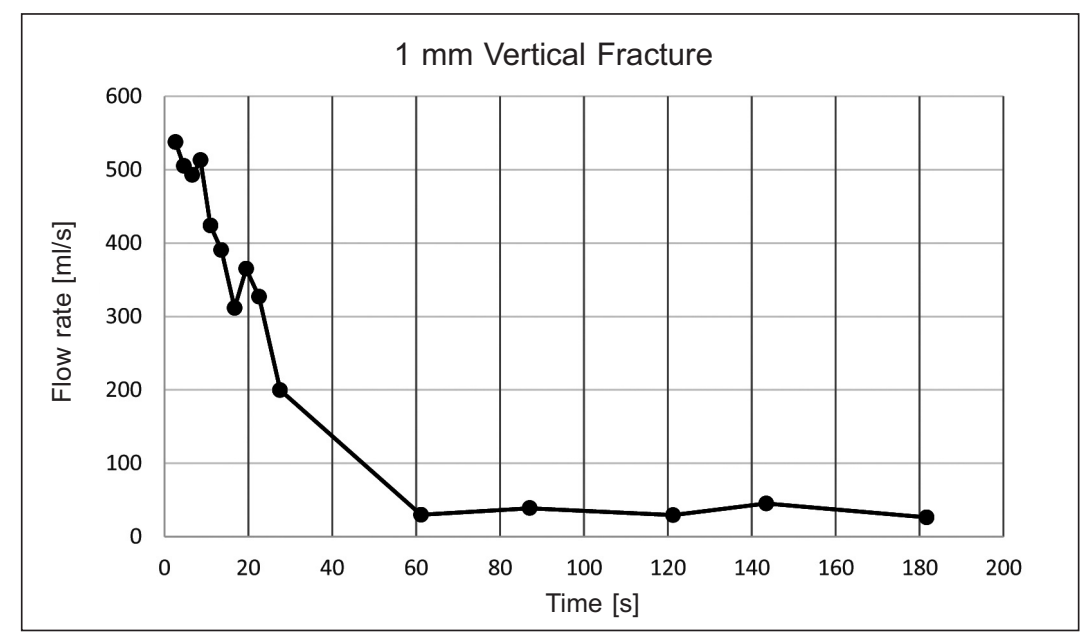

Fig. 12. Walnut at $10 \mathrm{~kg} / \mathrm{m}^{3}$ with size about $1 / 3$ of the fracture aperture 


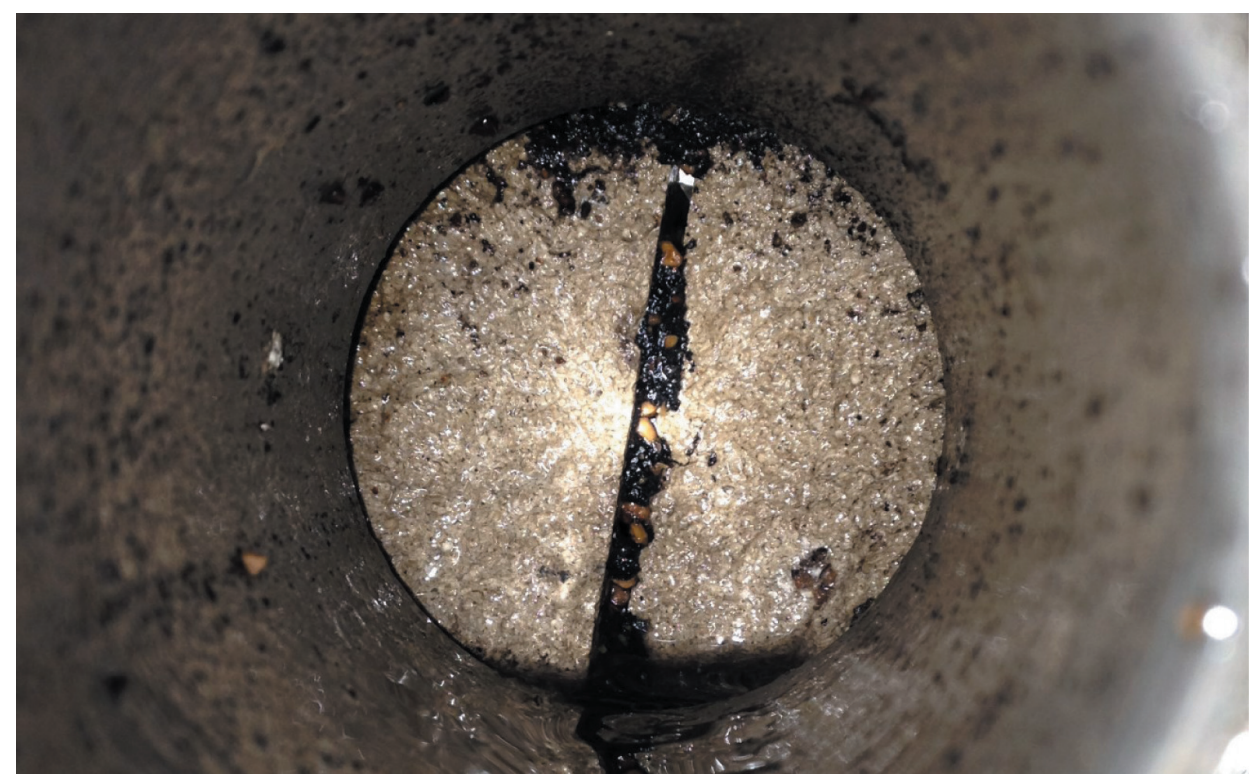

Fig. 13. Fracture opening $(1 \mathrm{~mm})$ partially plugged

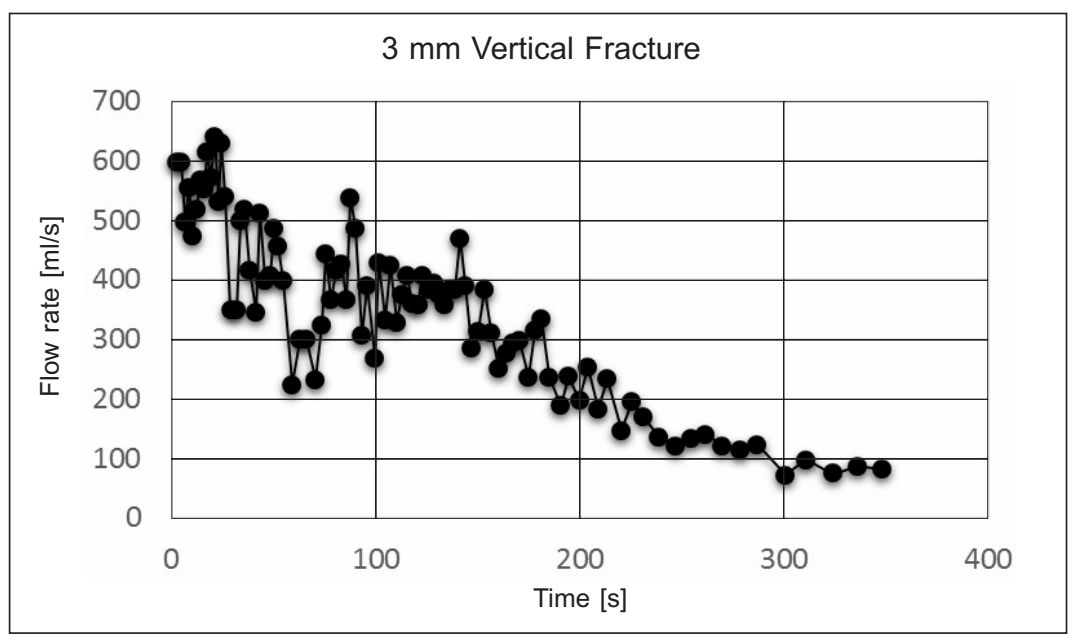

Fig. 14. Walnut at $20 \mathrm{~kg} / \mathrm{m}^{3}$ with size about $1 / 3$ of the fracture aperture

Figure 14 shows the performance of LCM having $20 \mathrm{~kg} / \mathrm{m}^{3}$ concentration in plugging a $3 \mathrm{~mm}$ vertical fracture. Once more, the flow rate presented reductions, but did not completely stop. Figure 13 shows that the lower parts of the fracture were sealed, while the top remained open. The comparison of the Figures 13 and 15 showed that the smallest fracture size resulted in a better plug in both cases. 


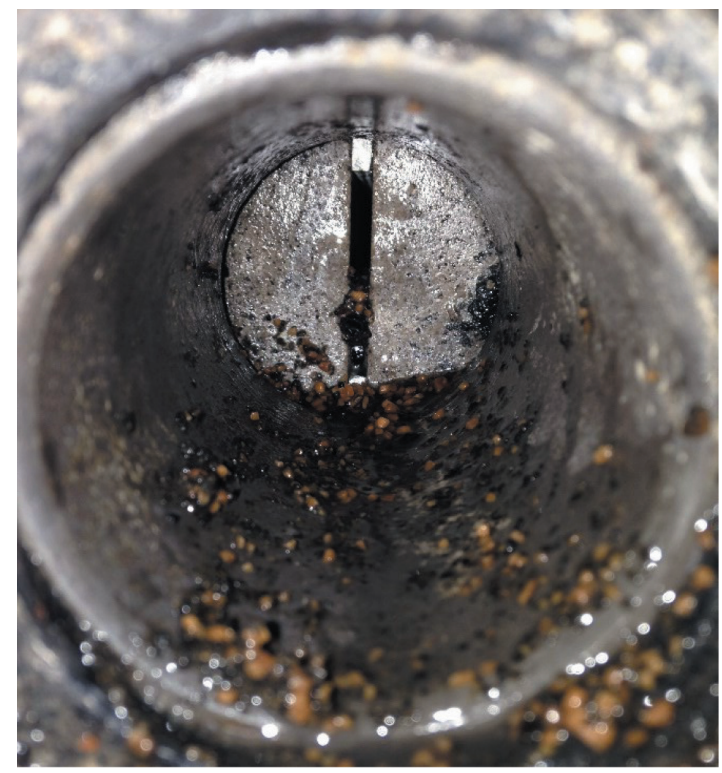

Fig. 15. Fracture opening $(3 \mathrm{~mm})$ partially plugged

\section{MODEL VERIFICATION}

The computer program described above allows users to make predictions on how long it takes for each particle size of LCM to plug a fracture. Furthermore, it also forecasts the flow rate after the LCM seal is formed.

A comparison between the results provided by the computer simulator and the experimental work is showed in Figures (9-11) 16-18.

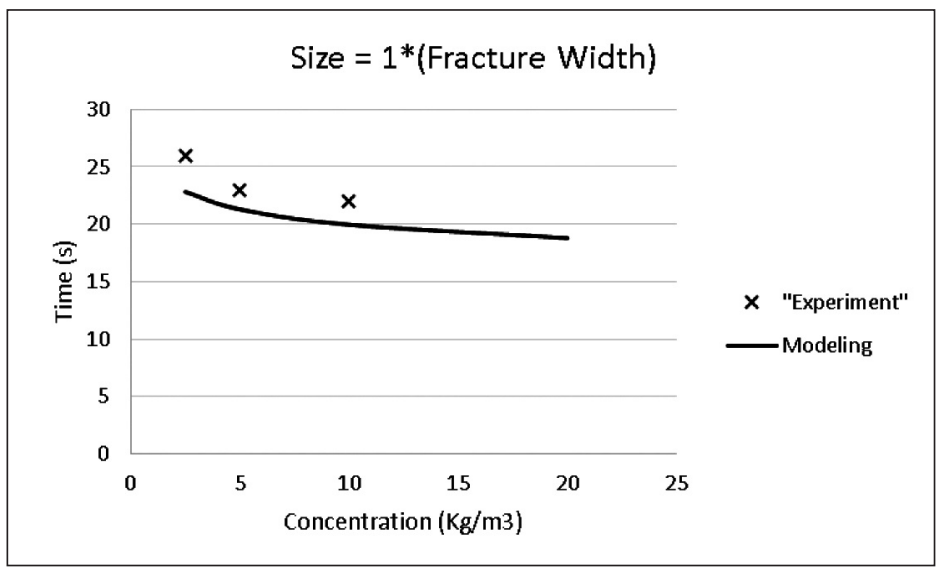

Fig. 16. Comparison: fracture is $1 \mathrm{~mm}$ and LCM is the same size 
Figure 16 shows that when the size of the particles of LCMs are about the same size of the fracture, the model slightly under predicted for a $1 \mathrm{~mm}$ fracture aperture.

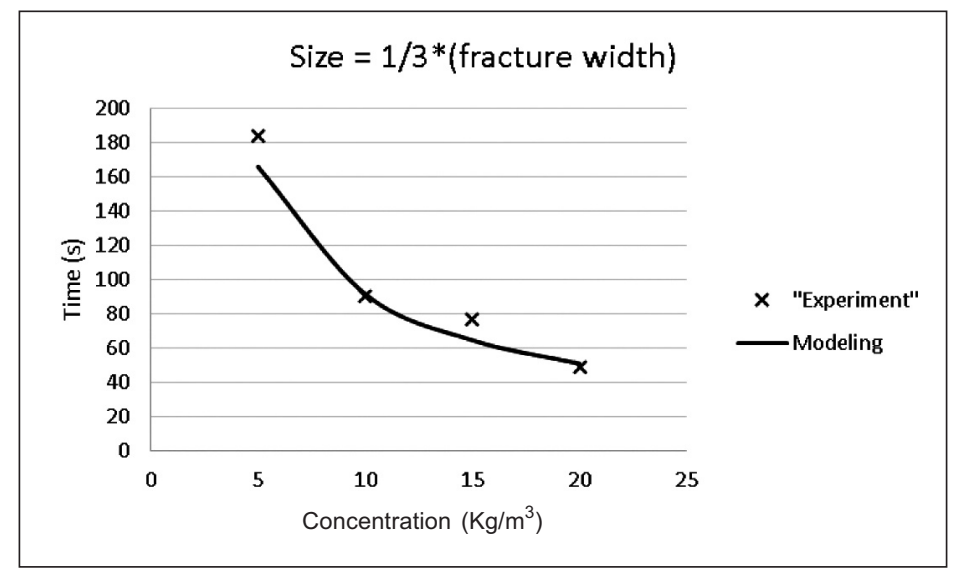

Fig. 17. Comparison: fracture is $1 \mathrm{~mm}$ and LCM is $1 / 3$ of fracture

Figure 17 presents a graph leading to the conclusion that the model is fairly accurate when the fracture is $1 \mathrm{~mm}$ and the sizes of LCMs particles are $1 / 3$ of the fracture aperture.

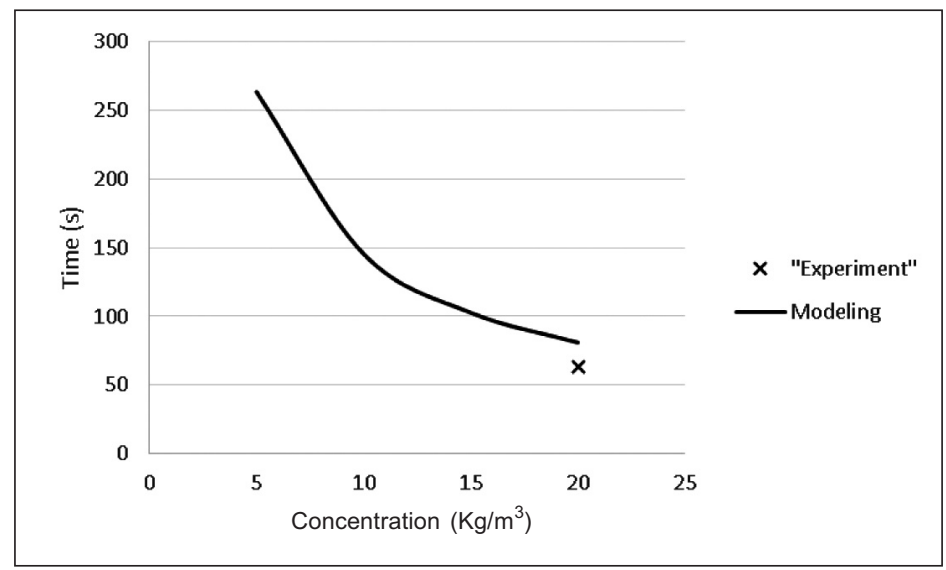

Fig. 18. Fracture is $3 \mathrm{~mm}$ and LCM is $1 / 3$ of fracture

For the case of a fracture opening as large as $3 \mathrm{~mm}$, Figure 18 shows that the model has a tendency to over predict the plug time; however, more experiments would be necessary to account for different concentrations. For $10 \mathrm{~kg} / \mathrm{m}^{3}$ the experiments, contrary to 
the model, showed no plug; nevertheless, the model predicted that a plug would be formed in about 2.5 minutes after the experiment started. Before that, the amount of fluid lost had already exceeded the capacity of the experimental setup.

\section{CONCLUDING REMARKS}

The model of the fractability of the LCM seal can be used to analyze the pressures that would make the seal break. These breakdown pressures were higher than the differential pressure that created the lost circulation in the beginning; nonetheless, sudden induction of surge and swab pressures inside the well may be enough to destroy the seal afterwards. In addition, according to this model, Young's Modulus does not affect the resistance of the seal. Moreover, the higher the Poison's ratio, the higher the resistance of the LCM filter cake.

1. The D90 of the LCM particles needs to be a value close to the width of the fracture aperture to reduce plug time.

2. The most accurate LCM performance was accounted for when the D50 was $1 / 3$ of the fracture opening, given the concentrations studied.

3. The concentration of LCM varies with fracture aperture and LCM size. Although $10 \mathrm{Kg} / \mathrm{m}^{3}$ of LCM was enough to plug a $1 \mathrm{~mm}$ fracture, it was too low for a $2 \mathrm{~mm}$ fracture. In the last case, $20 \mathrm{Kg} / \mathrm{m}^{3}$ had to be used.

4. The simulations showed that LCM pills containing small particles require high concentrations to plug the fracture. By maintaining concentration constant, lower sizes will take longer to plug the fracture if they plug them at all.

5. An accurate estimation of the fracture opening is the starting point for the proper choice of LCM. The quality of the seal will be as solid as the data concerning fracture geometry and bridge parameters such as Biot's coefficient, Poisson's ratio, and porosity. Those can be estimated in a surface lab.

6. A simulation using the computer program presented here is recommended prior to LCM application in the field. The program provides information about the PSD that should be employed. Furthermore, the simulation giver time estimation of when the fracture is plugged.

\section{Acknowledgment}

We wish to express our appreciation to The Tulsa University Drilling Research Projects for sponsoring this project. Many thanks are extended to Dr. Nicholas Takach for his editorial guidance. 


\section{REFERENCES}

[1] Alsaba M., Nygaard R.: Laboratory Evaluation of Sealing Wide Fractures Using Conventional Lost Circulation Materials. SPE Annual Technical Conference and Exhibition, Amsterdam, The Netherlands, October 27-29, 2014, SPE 170576-MS.

[2] Alsaba M., Nygaard R.: Lost Circulation Materials Capability of Sealing Wide Fractures. SPE Deepwater Drilling and Completions Conference, Galveston, TX, USA. September 10-11, 2014, SPE 170285-MS.

[3] Ivan C., Burton J., Bloys B.: Lost Circulation Can Be Managed Better Than Ever. World Oil, June, 2003.

[4] Liétard O., Unwin T., Guillot, D., et al.: Fracture Width Logging While Drilling and Drilling Mud/Loss-Circulation-Material Selection Guidelines in Naturally Fractured Reservoirs. SPEDC, vol. 14, No. 3, SPE 57713.

[5] Majidi R.: Modeling of Yield-Power-Law Type of Drilling Fluid Losses in Naturally Fractured Reservoirs. TUDRP Advisory Board Meeting Report, The University of Tulsa, Tulsa, Oklahoma, 2009.

[6] Mitchell R., Miska S.: Fundamentals of Drilling Engineering. Richardson, TX, Society of Petroleum Engineers, 2011.

[7] White R.: Lost-Circulation Materials and Their Evaluation. Spring Meeting of the Pacific Coast District, Division of Production, Los Angeles, USA, May, 1956.

[8] Whitfill D., Wang H.: Making Economic Decisions to Mitigate Lost Circulation. SPE Annual Technical Conference and Exhibition, Dallas, TX, USA, October 9-12, 2005, SPE 95561.

[9] Whitfill D., Wang M., Jamison D., Rogers A.: Preventing Lost Circulation Requires Planning Ahead. SPE International Oil Conference and Exhibition, Veracruz, Mexico, June 27-30, 2007, SPE 108647.

[10] Zeng H.: Study of Effectiveness of Lost Circulation Materials. TUDRP Advisory Board Meeting Report, The University of Tulsa, Tulsa, Oklahoma, 2014. 